\title{
Numerical modeling of flow around a heated cylinder with a rough surface
}

\author{
Lenka Lausová ${ }^{1, *}$, Vladimíra Michalcová ${ }^{1}$ and Ivan Kološ ${ }^{1}$ \\ ${ }^{1}$ VŠB - Technical University of Ostrava, Faculty of Civil Engineering, L. Podéště 1875/17, 70800 \\ Ostrava-Poruba, Czech Republic
}

\begin{abstract}
The article deals with the numerical solution of the load of a heated chimney from wind effects. The paper examines flow around a heated cylinder with the rough surface in high Reynolds number regime. The results of drag coefficient, pressure coefficient and other related flow properties are compared with the calculations of the flow around the unheated cylinder.
\end{abstract}

\section{Introduction}

One of the important topics in civil engineering is determining the load from the effect of the wind. It can be caused by the flow around obstacle, which can be a circular cylinder (smokestack) [1], square cylinder, and other types of objects for example [2-4]. Since the last century, it has been largely experiments that have made a significant contribution to the elucidation of the effect of flow past the circular cylinder $[1,5,6]$. In recent years, numerical modeling has become increasingly important, which is related to the ever-improving computer technology and results of different simulations can be found for example in publications [7-9].

A significant role in the loading of structures from the wind have not only the Reynolds number but also the roughness of the obstacle surface. Experimental study about the effects of surface roughness on the flow past circular cylinders is solved in [10]. Measurements of mean-pressure distributions and boundary-layer development on rough-walled circular cylinders describes [11]. The influence of surface roughness on the flow field of the cylinder can also be found in [12] for various type of roughness and values of Re numbers. Another broad issue is the study of the effect of a heated circular cylinder on the flow field at different Reynolds numbers, which is solved in [13] or [14]. The wake behind a heated cylinder was experimentally studied in [15]. Not many authors have been concerned with the influence of roughness on flow field characteristics of a heated cylinder [16] and [17].

The authors of this article have been focused on numerical simulations of the problem of the wind effect to a cylinder (chimney) with a rough sheathing [18] and [19]. They pointed out the sensitivity to small variations of affecting parameters in the critical regime. The objective of this comparative study is to use the results of the flow field of the cylinder with a rough surface at $15^{\circ} \mathrm{C}(288.15 \mathrm{~K})$ obtained in [19] for comparison with the selected flow field quantities of the identical cylinder heated by $\Delta T=150 \mathrm{~K}\left(150^{\circ} \mathrm{C}\right)$.

\footnotetext{
* Corresponding author: lenka.lausova@vsb.cz
} 


\section{Description of the task}

The task is focused on modeling of flow around the heated chimney with a corrugated sheet casing. This is a comparative study of flowing past a cylinder with a rough surface, where the first task is solved without the influence of temperature [19] and in the second task the cylinder is warmed by $\Delta T=150 \mathrm{~K}$. The study is performed in 2D solved by ANSYS Fluent software using SST $k$ - $\omega$ (Shear Stress Transport) turbulence model. Flow parameters in the atmospheric boundary layer correspond to highly turbulent flow. In the study, wind velocity is considered $u_{x}=10 \mathrm{~m} / \mathrm{s}$ and turbulence intensity $i_{u}=10 \%$. Reynolds number $\operatorname{Re}=2.1 \cdot 10^{6}$. Diameter of the chimney is $3.6 \mathrm{~m}$ and the height of the waves is $18 \mathrm{~mm}$. Further parameters are listed in Table 1.

Table 1. Parameters of the computational area and boundary conditions.

\begin{tabular}{|l|l|}
\hline Geometric dimensions & \multicolumn{1}{|c|}{ Values } \\
\hline Length of the area & $L=140 \mathrm{~m}$ \\
\hline Width of the area & $B=50 \mathrm{~m}$ \\
\hline Inner chimney diameter & $D=3.6 \mathrm{~m}$ \\
\hline Height of the waves & $h=18 \mathrm{~mm}$ \\
\hline $\begin{array}{l}\text { Considered surface } \\
\text { roughness }\end{array}$ & $h / D=5 \cdot 10^{-3}[-]$ \\
\hline $\begin{array}{l}\text { Distance of the chimney } \\
\text { axis from inlet }\end{array}$ & $l=25 \mathrm{~m}$ \\
\hline
\end{tabular}

\begin{tabular}{|l|l|}
\hline \multicolumn{1}{|c|}{$\begin{array}{c}\text { Parameters } \\
\text { of calculations }\end{array}$} & \multicolumn{1}{c|}{ Values } \\
\hline Air velocity & $u_{x}=10 \mathrm{~m} / \mathrm{s}$ \\
\hline Turbulence intensity & $i_{u}=10 \%$ \\
\hline Kinematic viscosity & $v=1.7 \cdot 10^{-5} \mathrm{~kg} /(\mathrm{m} \cdot \mathrm{s})$ \\
\hline Reynolds number & $\mathrm{Re}=2.1 \cdot 10^{6}[-]$ \\
\hline $\begin{array}{l}\text { Air density at the } \\
\left.\text { entrance (for } 15^{\circ} \mathrm{C}\right)\end{array}$ & $\rho=1.225 \mathrm{~kg} / \mathrm{m}^{3}$ \\
\hline Temperature increase & $\Delta T=150 \mathrm{~K}$ \\
\hline Time step & $0.005 \mathrm{~s}$ \\
\hline
\end{tabular}

\section{Description of numerical simulation}

In addition to the Continuity equation (1) and the Navier-Stokes equation (2), the Energy equation (3) is solved in this task [20]. The equation (3) represents the energy transfer due to conduction and viscous dissipation. Results are the following variables: flow velocity $\vec{u}[\mathrm{~m} / \mathrm{s}]$, pressure $p[\mathrm{~Pa}]$ and temperature $T[\mathrm{~K}]$.

$$
\begin{gathered}
\frac{\partial \rho}{\partial t}+\nabla \cdot(\rho \vec{u})=0 \\
\frac{\partial}{\partial t}(\rho \vec{u})+\nabla \cdot(\rho \vec{u} \vec{u})=-\nabla p+\nabla \cdot(\overline{\bar{\tau}})+\rho \vec{g}+\vec{F} \\
\frac{\partial}{\partial t}(\rho E)+\nabla \cdot[\vec{u}(\rho \mathrm{E}+p)]=\nabla \cdot(\lambda \nabla \mathrm{T})-\nabla \cdot(\overline{\bar{\tau}} \cdot \vec{u})
\end{gathered}
$$

In the equations (1)-(3) $\overline{\bar{\tau}}$ represents stress tensor expressing friction forces (4), $\vec{g}$ gravitational and $\vec{F}$ other external volume forces, $\rho\left[\mathrm{kg} / \mathrm{m}^{3}\right]$ air density, $T[\mathrm{~K}]$ air temperature, $\lambda[\mathrm{W} /(\mathrm{m} \cdot \mathrm{K})]$ thermal conductivity.

$$
\overline{\bar{\tau}}=\mu\left[\left(\nabla \vec{u}+\nabla \vec{u}^{T}\right)-\frac{2}{3} \nabla \cdot \vec{u} \cdot I\right]
$$

In the equation (4) $\mu[\mathrm{Pa} \cdot \mathrm{s}]$ is dynamic viscosity and $I$ is the unit tensor.

Fluid properties may vary significantly with temperature. The air density $\rho$ is constant in isothermal flow, $\rho=1.225 \mathrm{~kg} / \mathrm{m}^{3}$ for temperature $288.15 \mathrm{~K}\left(15^{\circ} \mathrm{C}\right) . \quad \rho$ for incompressible non-isothermal flow is calculated automatically from the gas state equation at constant 
operating pressure $p_{0}=100 \mathrm{kPa}$. The parameters entering into the numerical solution in the equations depended upon the actual air temperature $T[\mathrm{~K}]$ are thermal conductivity $\lambda[\mathrm{W} /(\mathrm{m} \cdot \mathrm{K})]$, specific heat capacity of the air $c_{\mathrm{p}}[\mathrm{J} /(\mathrm{kg} \cdot \mathrm{K})]$, dynamic viscosity $\mu[\mathrm{Pa} \cdot \mathrm{s}]$.

The RANS model SST $k-\omega$ is chosen for the numerical solution as the most suitable model for the task where the aim of the investigation is the influence of surface roughness on the turbulent quantities [19].

Mesh consists of 120,000 elements, the boundary layer is from quadrilateral elements in 6 prismatic layers, the rest of the mesh is formed by triangular cells. Detail of thickened mesh in the domain and around the waves on the cylinder surface is displayed in Fig. 1 [19].
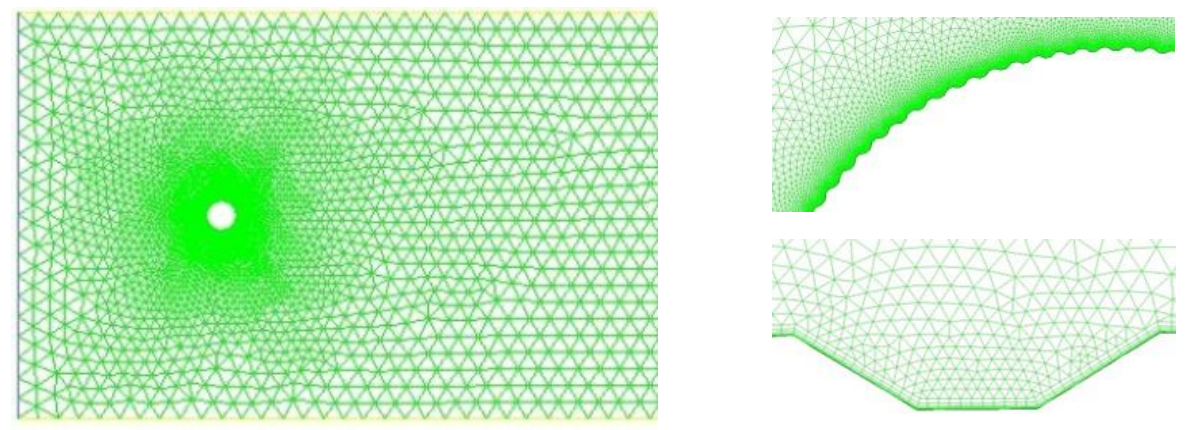

Fig. 1. Meshing - thickened mesh in the domain (left) and detail of thickened mesh around the waves on the cylinder surface (right).

\section{Results from numerical solution}

Comparison of the numerical results of both heated ("Therm") and unheated ("No therm") cylinder is performed for these variables: pressure coeficient $c_{p}$ around the circumference of the cylinder, fluctuation of $c_{p}$ around the circumference of the cylinder, difference in frequency of vortex shedding and the value of drag coeficient $c_{d}$.

The positions of the points in which the pressure load is monitored in more detail are indicated in Fig. 2. The individual points on the circumference of the cylinder are divided into several categories: internal points, external points, points in the center of the waves, which are also divided into "uphill" and "downhill", the points of all categories were defined in each wave of the surface roughness.
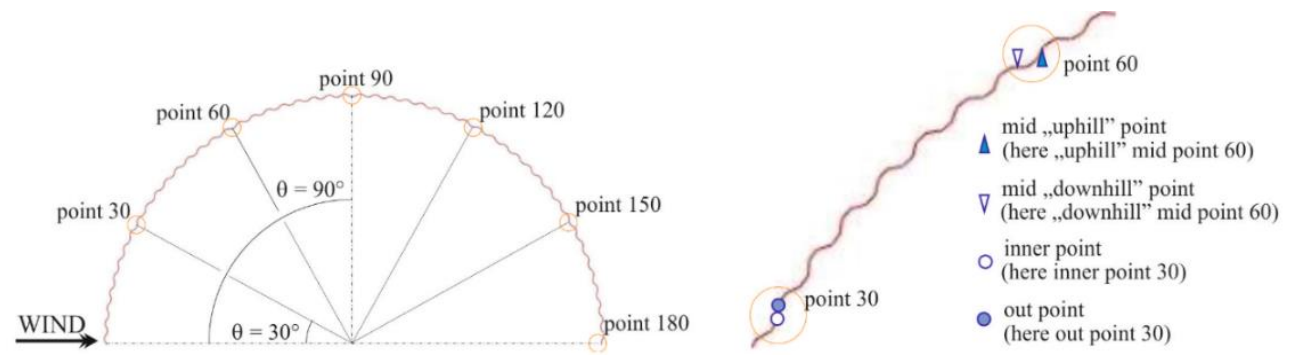

Fig. 2. Monitored points on the surface of the cylinder [19].

The division of midpoints into two categories "uphill" and "downhill" is due to their different microclimate in the immediate vicinity of the cylinder. They differ from one another in the direction of the wave slope with respect to the air flow (one forms a windward micro side and the other a leeward side). This is also shown by the swirling flow field in the 
immediate vicinity of the cylinder in Fig. 3, which is due to the significant roughness of its surface.

\section{NO THERM}

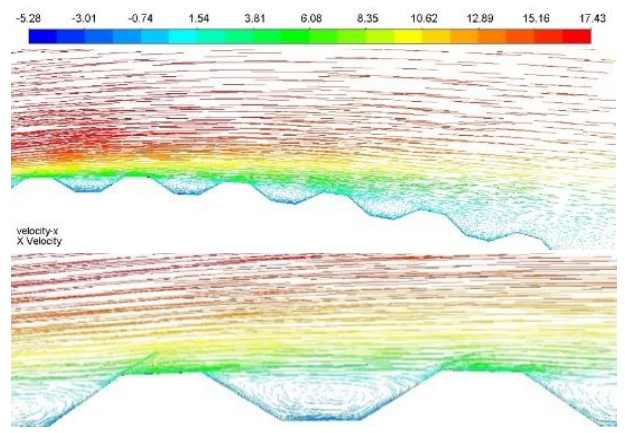

THERM

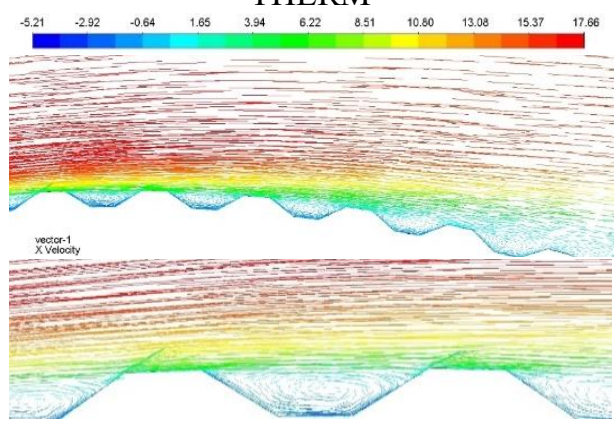

Fig. 3. Swirling flow field in the immediate vicinity of the unheated (left) and heated cylinder (right).

\subsection{Mean pressure coefficient}

The pressure load at the circumference of the surface roughness cylinder is evaluated by a dimensionless mean pressure coefficient $\bar{c}_{p}$, for the constant air density $\rho$ is defined:

$$
\bar{c}_{p}=\frac{\bar{p}_{i}}{\bar{p}_{d y n}}=\frac{\bar{p}_{c i}-\bar{p}_{r e f}}{1 / 2 \cdot \rho \cdot \bar{u}_{r e f}^{2}}
$$

The reference point is determined at the horizontal axis of the cylinder at a distance of $20 \mathrm{~m}$, that is, about 5.55 D in front of its vertical axis. In the equation (5) the $\bar{p}_{\text {ref }}[\mathrm{Pa}]$ is mean static pressure at the reference point, $\bar{p}_{c i}[\mathrm{~Pa}]$ is the mean resulting static pressure on the cylinder surface at the $i$-point, $\bar{u}_{r e f}[\mathrm{~m} / \mathrm{s}]$ is mean stream wise velocity at the reference point.

The $\bar{c}_{p}$ records of midpoints uphill and downhill separately for unheated and heated task are in Fig. 4. It can be seen from the record that heating of the cylinder at a given wind velocity almost does not affect the load. The position of the point with the minimum load is the same for uphill points, for the downhill the minimum slightly shifted to the left. On the back of the cylinder, from an angle of $\theta=120^{\circ}$, the load due to heating slightly increased.
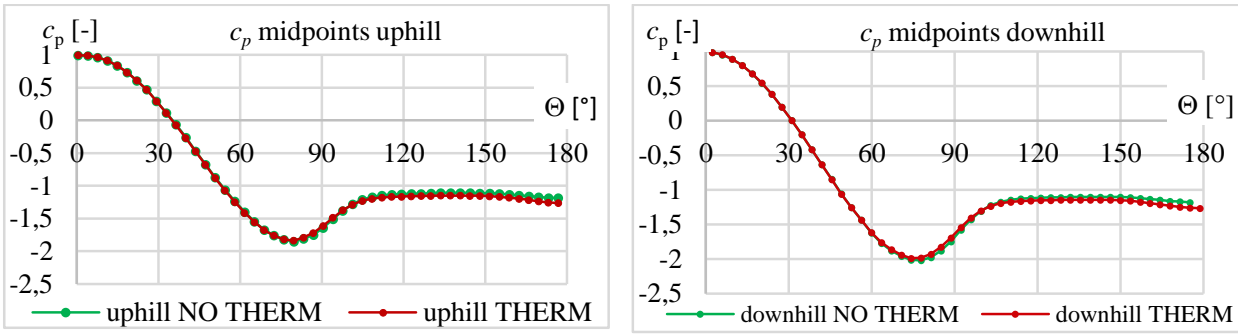

Fig. 4. $c_{p}$ comparison in points uphill (left) and downhill (right) for unheated and heated cylinder.

In Fig. 5 there is a record of $\overline{c_{p}}$ at midpoints ,uphill“ and „downhill“ separately for „No therm“ and „Therm“ task which proves the findings mentioned above. 

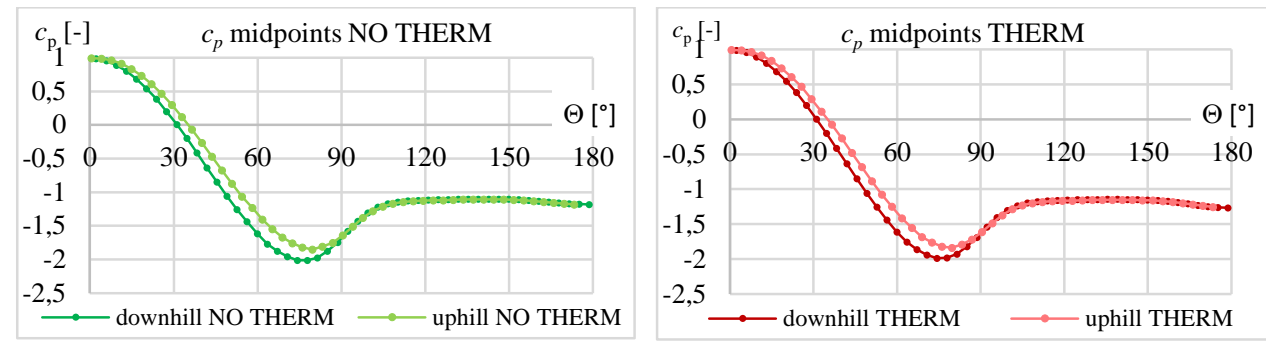

Fig. 5. $c_{p}$ comparison in downhill and uphill points for unheated (left) and heated (right) cylinder.

\subsection{Fluctuation of pressure coefficient around the circumference}

In the presented work, fluctuation of the pressure coefficient $c_{p}$ is defined by the dimensionless mean pressure fluctuations coefficient $c_{p, r m s}$, which is given by (6). The courses $c_{p, r m s}$ around the circumference of the heated and unheated cylinder calculated at all monitored points are displayed in Fig. 6 and Fig. 7.

$$
c_{p, r m s}=\frac{p_{r m s}}{p_{d y n}}=\frac{\left(p_{c i}^{2} / \bar{p}_{c i}-p_{r e f}^{2} / \bar{p}_{r e f}\right)}{1 / 2 \cdot \rho \cdot u_{r e f}^{2}}
$$

In the equation (6) $p_{r m s}$ is mean static pressure fluctuations [Pa].
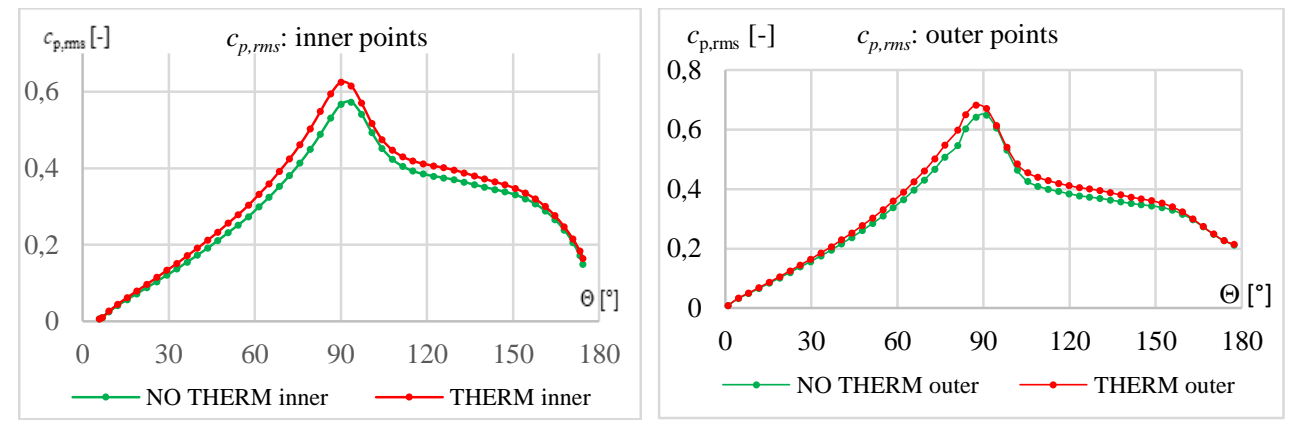

Fig. 6. $c_{p, r m s}$ of heated and unheated cylinder in inner (left) and outer (right) points.
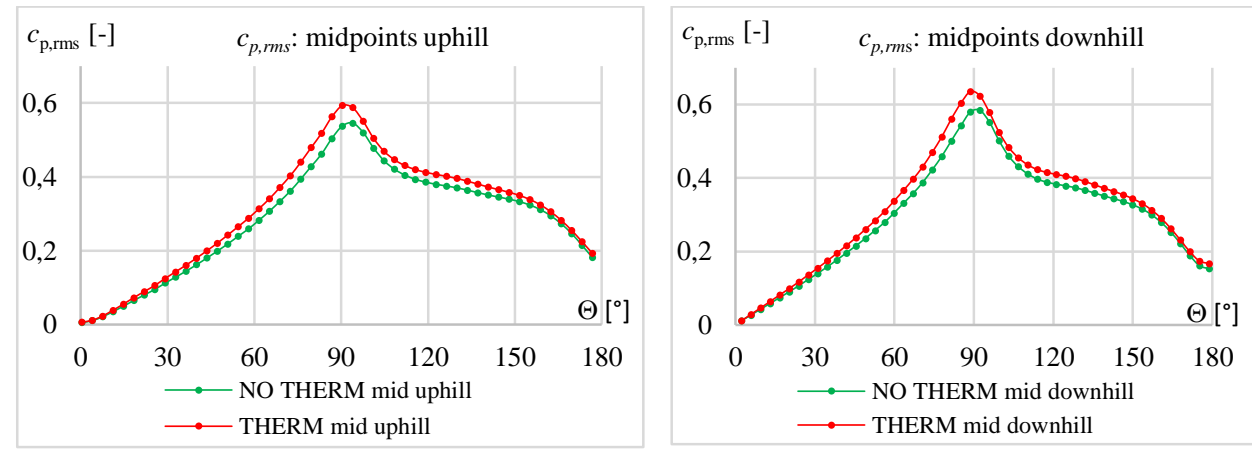

Fig. 7. $c_{p, r m s}$ of the heated and unheated cylinder in uphill (left) and downhill midpoints (right).

Higher fluctuation of instantaneous pressure loads is evident at all points. This is consistent with the velocity vectors in Fig. 3, which show (insignificantly) a denser swirling 
flow field around the heated cylinder. In both cases ("Therm" and "No therm") and in all "downhill" and "uphill" points the fluctuations of extreme values are reached in the vertical axis of the cylinder where there is also the biggest difference.

\subsection{The frequency of vortex shedding: Strouhal number}

Vortex shedding in the wake of the cylinder is dictated by the Strouhal number St (7).

$$
\mathrm{St}=\frac{f \cdot D}{u_{x}}
$$

In the equation (7) $f$ is frequency of vortex shedding $\left[\mathrm{s}^{-1}\right]$, circular diameter $D=3.6 \mathrm{~m}$ (inner diameter), air velocity $u_{x}=10 \mathrm{~m} / \mathrm{s}$.

St number is difficult to define for presented $\mathrm{Re}=2.1 \cdot 10^{6}$. Conclusions of experiments for cylinders with surface roughness are not yet available in a comprehensive form [19]. The Strouhal number for described task was determined based on the lift coefficient $c_{1}$ and the subsequent recalculation of the frequency of vortex shedding $f$. The values obtained from the numerical simulations are displayed in Table 2.

Table 2. Strouhal number.

\begin{tabular}{|l|c|c|}
\hline & NO THERM & THERM \\
\hline Average time of one period of vortex shedding [s] & 1.499 & 1.513 \\
\hline Frequency of one period of vortex shedding $f[1 / \mathrm{s}]$ & 0.667 & 0.662 \\
\hline Calculated Strouhal number St [-] & 0.240 & 0.238 \\
\hline
\end{tabular}

Fig. 8 is a 10 -second record of lift coefficient. The record shows a slight shortening of the frequency for one period of the vortex shedding $f$ and thus a decrease in the value of St number, and also a small asymmetry in the oscillation of the heated cylinder. The value of lift coefficient varies for unheated cylinder in the interval $\langle 1 ;-1\rangle$, and for the heated cylinder in the interval $\langle 1.05 ;-1.11\rangle$.

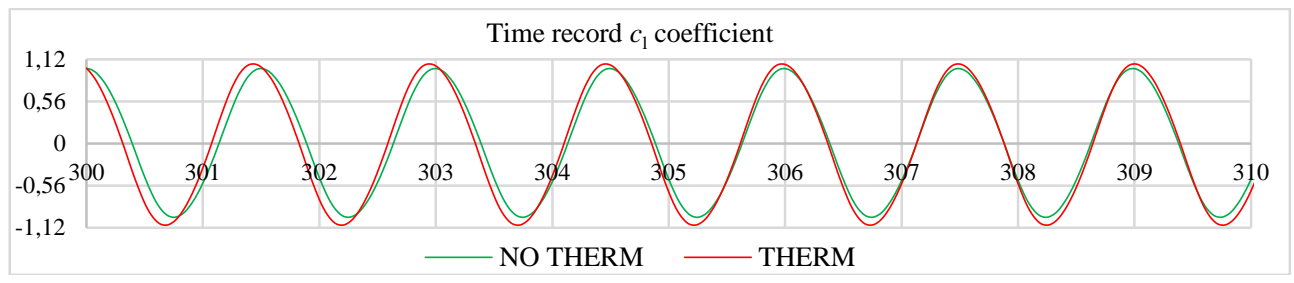

Fig. 8. Time record - lift coefficient $c_{1}$.

\subsection{Drag coefficient - comparison with standard calculation}

The standard solution of chimney load is based on the drag coefficient $c_{d}$ (the force coefficient $\left.c_{f, 0}\right)$. Its determination according to the valid standard EN 1991-1-4 takes into account only the air flow around the cylinder with a rough surface, which represents only a small height of unevenness and does not take into account their shape. Table 3 shows $c_{d}$ values from numerical simulations determined according to the EN 1991-1-4 for described process of an unheated cylinder. Drag coefficient values obtained from numerical simulations for unheated cylinder are less than 1.0 [19], on the contrary, the value for a heated cylinder is $c_{d}=1.04$. 
Table 3. Value of drag coefficient $c_{d}$.

\begin{tabular}{|l|c|c|c|}
\hline & Standard calculation & Unheated cylinder & Heated cylinder \\
\hline$c_{d}$ coefficient $[-]$ & 1.01 & 0.99 & 1.04 \\
\hline
\end{tabular}

\section{Conclusions}

The work presents the role of numerical modeling of flow around a circular cylinder with a rough surface (smokestack). The 2D task simulates a highly turbulent flow corresponding to the real atmosphere. The calculations are carried out in the ANSYS Fluent software using the SST $k$ - $\omega$ model, which allows a better mathematical description near the wall compared to other RANS models. The study is focused on the calculation of the pressure load of the heated and unheated cylinder from the effect of the wind, which is represented here by the dimensionless coefficient $c_{p}$.

Since it is cylinder with a very rough surface, attention is also paid to the influence of the point location in surface roughness. From the structural load calculation point of view, the results show higher fluctuation of instantaneous pressure loads at all examined points of the surface of the cylinder heated by $\Delta T=150 \mathrm{~K}$. Record of the lift coefficient $c_{1}$ shows a slight shortening of the frequency of one period of the vortex shedding $f$ and thus a decrease in the value of St number, and also a small asymmetry in the oscillation of the heated cylinder.

The $c_{d}$ coefficient time record is compared to the standard calculation, for the unheated cylinder the value of $c_{d}$ from numerical simulations is less than 1.0, for the heated cylinder, on the contrary, the result of $c_{d}$ value is bigger.

Since the rate of heat transfer is strongly dependent on fluid velocity and it is possible to assume more significant differences in the resulting pressure on the cylinder surface for lower velocities, the authors will focus on a detailed analysis of the flow field of this task in 3D and also on calculations for other Re numbers (resp. velocities). The findings contribute to extend the study to modified surface roughness shapes and its influence on the flow field characteristics of the heated cylinder.

The paper has been supported by the project of "Conceptual development of science and research activities 2020" on the Faculty of Civil Engineering, VŠB - TU Ostrava and by the Ministry of Education, Youth and Sports from the Large Infrastructures for Research, Experimental Development and Innovations project „IT4Innovations National Supercomputing Center - LM2015070“.

\section{References}

1. M. M. Zdravkovich, Flow around Circular Cylinders: A Comprehensive Guide through Flow Phenomena, Experiments, Applications, Mathematical Models, and Computer Simulations. Vol. 2: Applications (2003)

2. N. Jendzelovsky, R. Antal, and L. Konecna, MATEC Web Conf. 107, 00081 (2017)

3. M. Macak, O. Hubova, and L. Konecna, 16th Conf. Appl. Math. APLIMAT 2017 Proc. 941-951 (2017)

4. K. Kotrasova and E. Kormanikova, Adv. Math. Phys. 2017, 1-9 (2017)

5. C. Norberg, J. Fluids Struct. 17, 57-96 (2003)

6. C. Norberg, in IUTAM Symp. Bluff-Body Wakes, Dyn. Instab. (Springer Berlin Heidelberg, Berlin, Heidelberg, 275-278, 1992)

7. I. Rodríguez, O. Lehmkuhl, R. Borrell, L. Paniagua, and C.D. Pérez-Segarra, Procedia Eng. 61, 166-172 (2013) 
8. X. X. Cheng, L. Zhao, Y. J. Ge, J. Dong, and C. Demartino, Wind Struct. 24, 119144 (2017)

9. A. C. Benim, M. Cagan, A. Nahavandi, and E. Pasaqualotto, Proc. 5th IASME / WSEAS Int. Conf. Fluid Mech. Aerodyn. 232-237 (2007)

10. Ribeiro, (n.d.)

11. O. Güven, C. Farell, and V. C. Patel, J. Fluid Mech. 98, 673-701 (1980)

12. A. Benidir, O. Flamand, L. Gaillet, and G. Dimitriadis, J. Wind Eng. Ind. Aerodyn. 137, 1-13 (2015)

13. S. J. Quintavalla, A. J. Angilella, and A. J. Smits, Exp. Therm. Fluid Sci. 48, 15-18 (2013)

14. P. H. Oosthuizen and S. Madan, J. Heat Transfer 93, 240-242 (1971)

15. M. Khashehchi, I. Ashtiani Abdi, and K. Hooman, Int. J. Heat Mass Transf. 86, 589-599 (2015)

16. E. Achenbach, Int. J. Heat Mass Transf. 20, 359-369 (1977)

17. F. Dierich and P.A. Nikrityuk, Int. J. Therm. Sci. 65, 92-103 (2013)

18. V. Michalcova and L. Lausova, Comput. Struct. (2017)

19. I. Kološ, V. Michalcová, and L. Lausová, Math. Methods Appl. Sci. mma.5901 (2019)

20. ANSYS Fluent Theory Guide, Release 19.2 (ANSYS, Inc., 2018) 